XIAONIAN SHAN, Ph.D. Candidate ${ }^{1}$

E-mail: 3ashan@tongji.edu.cn

JIANHONG YE, Ph.D. ${ }^{1}$

(Corresponding author)

E-mail: jianhong_ye@tongji.edu.cn

XIAOHONG CHEN, Ph.D. ${ }^{1}$

E-mail: chenxh@tongji.edu.cn

${ }^{1}$ Tongji University, Key Laboratory of Road and Traffic

Engineering of the Ministry of Education

No. 4800, Cao'an Road, Shanghai, 201804, China
Human - Transport Interaction

Preliminary Communication

Submitted: Sep. 14, 2015

Accepted: July 14, 2016

\title{
PROPOSING A REVISED PEDESTRIAN WALKWAY LEVEL OF SERVICE BASED ON CHARACTERISTICS OF PEDESTRIAN INTERACTIVE BEHAVIOURS IN CHINA
}

\begin{abstract}
The objective of this study is to analyse characteristics of Pedestrian Interactive Behaviours (PIBs) in order to propose a revised pedestrian walkway Level of Service (LOS) in China. Field data on overtaking and evasive behaviours were collected at a metro station walkway in Shanghai, China to calculate macro and micro indicators. Occurrence intensities of these two PIBs initially increased with moderate density and later decreased with high density that reduced available space. PIBs were also analysed in terms of sideways behaviours to account for the varying difficulties of PIBs at different densities. It was found that available space for PIBs was the main factor contributing to the intensity features. Moreover, the different space demands of the two PIBs resulted in different features between them. Finally, a revised pedestrian walkway LOS was proposed based on the macro and micro characteristics of PIBs in China.
\end{abstract}

\section{KEY WORDS}

pedestrian level of service; overtaking behaviour; evasive behaviour; macro and micro characteristics; metro station walkway;

\section{INTRODUCTION}

During the process of pedestrians moving in clusters, common interactive behaviours will inevitably happen, such as overtaking behaviour and evasive behaviour. The characteristics of these behaviours at different densities are one key element that affects pedestrian flow and the ability of pedestrians to evacuate safely. These characteristics are also decisive indicators used to evaluate the Level of Service (LOS) of pedestrian flow.

Previously, studies regarding Pedestrian Interactive Behaviours (PIBs) including overtaking behaviour [1-5], evasive behaviour [6-8] and weaving behaviour [9-10] have been conducted on walkways. Previous studies mainly focused on two aspects of PIBs: (1) distances necessary for taking behaviours to occur (micro level); (2) occurrence frequencies at a specific density (macro level). On the former subject, many studies have investigated the longitudinal distance between pedestrians before a behaviour occurs and the horizontal distance between pedestrians after the behaviour has occurred. Controlled experiments [1-3, 8-10, 11] and field observations [4, 7] were most often conducted to investigate these distances. For example, Wu et al. reported that the average longitudinal distance before overtaking behaviour occurs is $2.4 \mathrm{~m}$ and the horizontal distance after overtaking behaviour has occurred is about $0.75 \mathrm{~m}$ at low density [4]. Kim et al. found that the average longitudinal distance before evasive behaviour occurs is $0.65 \mathrm{~m}$, while the average horizontal distance after evasive behaviour has occurred is 0.49 $\mathrm{m}$ [7]. Ma et al. found that pedestrians keep a distance of about $0.4 \mathrm{~m}$ to the wall, and the average relaxation distance between pedestrians is about $1.08 \mathrm{~m}$ [11].

Other studies explored the occurrence frequencies of these behaviours, and as result they have illustrated the relationship between density and probability of conflict [4, 7, 12-14]. Fruin analysed the changing trend of probability of conflict with the increase of space, the inverse of density, to cross-flow traffic [12]. The Highway Capacity Manual (HCM) qualitatively describes the characteristics of pedestrian interactive behaviours at different levels of service. For instance, at LOS of C, a pedestrian will always change their walking direction or speed to avoid conflict [13-14]. Wu et al. analysed the occurrence frequency features of pedestrian overtaking behaviour in free flow with the range of density less than $0.3 \mathrm{p} / \mathrm{m}^{2}$ [4]. Kim et al. established a multiple regression model to calculate the total number of evasive movements by using the two main influence factors: pedestrian volume and sidewalk width. With their results, Kim et al. also proposed a new measure of pedestrian LOS that incorporates 
evasive movements [7]. What is more, pedestrian simulation models have been revised to reflect the effect of PIBs, such as social force model [15] and multiagent distinct element method model [16].

After reviewing previous studies, it was found that they have the following issues: (1) previous field data were collected in free-flowing pedestrian traffic with a shortage of high density data in field observations; (2) only a single behaviour was analysed without comparing two different behaviours; and (3) the relationship between the macro and micro features of PIBs was not well explored. Moreover, as the qualitative descriptions of pedestrian interactive behaviours' characteristics at different levels of service were presented in HCM 2010 [13], it is important to explain the veracity of these descriptions with quantitative research. A revised LOS should be proposed based on the characteristics of PIBs to reflect actual pedestrian walking conditions. Meanwhile, with the rapid development of rail transit in China, the revised LOS could be used to evaluate the walking conditions on metro station walkways.

Therefore, the objective of this study is to analyse the characteristics of pedestrian interactive behaviours in order to revise pedestrian LOS on metro station walkways. More specifically, this study includes the following three tasks: (1) to analyse the macro and micro features of PIBs with varying density on walkways, including overtaking behaviour and evasive behaviour; (2) to explore the relationship between occurrence frequency features and distance indicators; and (3) to propose a revised LOS on metro station walkways in China.

\section{DATA}

\subsection{Indicators definition}

Based on the objective of this study, the behaviour indicators were divided into two types. One type of indicators is called macro indicators which characterize the occurrence frequency, named intensity, and the proportion of sideways behaviour (squeezing past other people because of limited walking space) at a certain density; the other type of indicators is called micro distance indicators which illustrate the amount of space necessary for taking behaviours to occur at a specific density, as shown in Table 1 where $T_{i}$ is the amount of time in minutes at a certain density (i); $O_{i}$ is the number of times that overtaking behaviour occurs during the period $T_{i}$; $E_{i}$ is the number of times that evasive behaviour occurs during the period $T_{i} ; P(i)$ is the frequency of PIBs per minute; $S_{o i}$ is the number of times that sideways overtaking behaviour occurs during the period $T_{i} ; S_{e i}$ is the number of times that sideways evasive behaviour occurs during the period $T_{i} ; R(i)$ is the proportion of sideways behaviours to the total behaviours; $o$ is overtaking behaviour, and $e$ is evasive behaviour.

\subsection{Observation site}

To achieve the research aims, the following criteria were applied in the site selection process: (1) investigation segment should have level ground with continuous pedestrian flow and be free from interruption from bicyclists and motor vehicles; (2) pedestrian flow should be bidirectional in order to observe pedestrian evasive behaviour on the investigation segment; (3) segment has constant path width; and (4) pedestrian flow within the segment is varied and includes low, middle and high density, to observe the different levels of service.

A metro station named People Square Station (PSS) in Shanghai, China, was chosen as the observing site because of large amounts of pedestrian volume. PSS is a transfer hub for three rail transit lines. In 2009, daily passenger flow volumes of PSS including exchanging volumes were 580,000 person-times. During peak hours, passenger flow volumes reached nearly 20 percent of daily volumes. Thus, the PSS provides a perfect observation site for pedestrian flow at varying densities.

The chosen walkway to collect field data in PSS is shown in Figure 1. The width of this walkway is $4.41 \mathrm{~m}$, and the length is $6 \mathrm{~m}$. There are no disturbances or obstacles in the observation site. The pedestrian flow is smooth and continuous. The survey was conducted by recording videos of pedestrian traffic on this walkway during peak and off-peak hours. The observed time was 7:00-10:10 in the morning and 16:00-19:10 in the evening in order to observe the pedestrian flows of varying density.

Table 1 - Indicators to be analysed for PIBs

\begin{tabular}{||c|l|c||}
\hline \hline Level of indicators & \multicolumn{1}{|c|}{ Description of indicators } & Symbols and calculation formulas \\
\hline \hline \multirow{3}{*}{ Macro } & Frequency of pedestrian interactive behaviour per minute & $P_{o}(i)=O_{j} / T_{i}$ and $P_{e}(i)=E_{j} / T_{i}$ \\
\cline { 2 - 3 } & Proportion of sideways behaviour to the total behaviour & $R_{o}(i)=S_{o} / O_{i}$ and $R_{e}(i)=S_{e} / E_{i}$ \\
\hline \multirow{3}{*}{ Micro } & $\begin{array}{l}\text { Longitudinal distance between pedestrians before } \\
\text { a behaviour occurs }\end{array}$ & $L_{o}$ and $L_{e}$ \\
\cline { 2 - 3 } & $\begin{array}{l}\text { Horizontal distance between pedestrians after } \\
\text { the behaviour has occurred }\end{array}$ & $H_{o}$ and $H_{e}$ \\
\hline
\end{tabular}




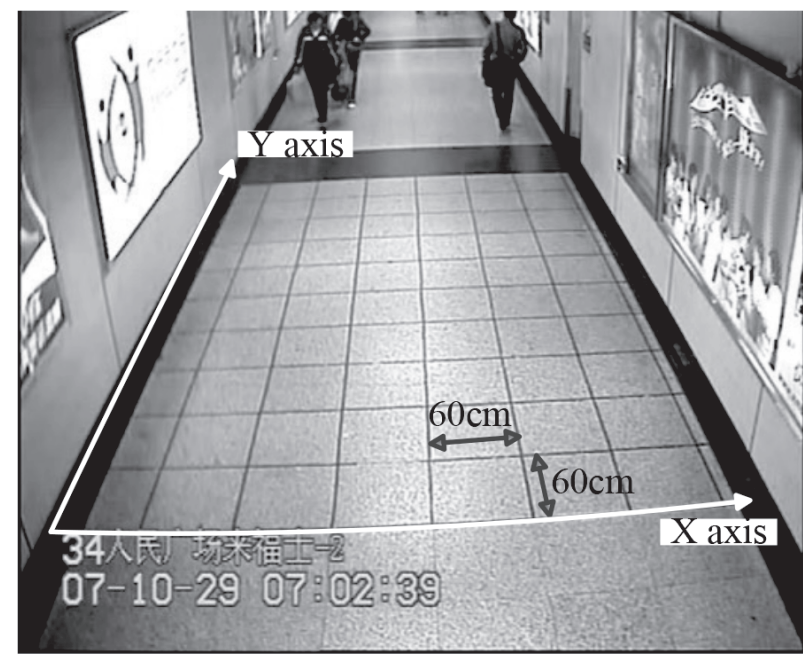

Figure 1 - The observed metro station walkway with grids

\subsection{Data extraction}

The data were extracted through hand counting. As shown in Figure 2, a pedestrian needs to change their walking direction before a behaviour occurs, and we can judge whether they take a sideways behaviour or not, after the behaviour has occurred. Thus, we can record all densities before PIBs occur by replaying videos and count the number of sideways behaviours at a certain density. It is notable that the observed time length at a certain density varied with densities. To solve this problem, the five seconds time interval densities were recorded to calculate the observed time length at a certain density. Therefore, the macro indicators could be calculated.

In order to measure the micro indicators of PIBs, the distance markings of $60 \times 60 \mathrm{~cm}$ squares were pasted on the tiles of the observed walkway in advance using dark tapes, and a rectangular coordinate system was established, as shown in Figure 1. Due to data collection by hand counting, it was necessary to identify whether the coordinates of relevant pedestrians could be easily collected or not before and after the behaviour occurs. To guarantee the veracity of pedestrians' coordinates, video image can be magnified to pedestrian's foot. The average foot breadth and length can be used as reference values to confirm the coordinates of pedestrians' feet. As can be seen in Figure 2, the relevant pedestrians' coordinates before an overtaking behaviour occurs are $\left(x_{01}, y_{01}\right)$ and $\left(x_{03}\right.$, $\left.y_{03}\right)$; the coordinates are $\left(x_{02}, y_{02}\right)$ and $\left(x_{03}, y_{03}\right)$ after the behaviour has occurred. Before an evasive behaviour occurs, the coordinates are $\left(x_{e 1}, y_{e 1}\right)$ and $\left(x_{e 3}\right.$, $\left.y_{\text {e3 }}\right)$; after the behaviour has occurred, the coordinates are $\left(x_{e 2}, y_{e 2}\right)$ and $\left(x_{e 4}, y_{e 4}\right)$. Then, the following formulas can be used to calculate the micro indicators:

To overtaking behaviour, $L_{0}=\left|y_{01}-y_{03}\right|, H_{0}=\left|x_{02}-x_{02}\right|$;

To evasive behaviour, $L_{e}=\left|y_{e 1}-y_{e 3}\right|, H_{e}=\left|x_{e 2}-x_{e 2}\right|$.

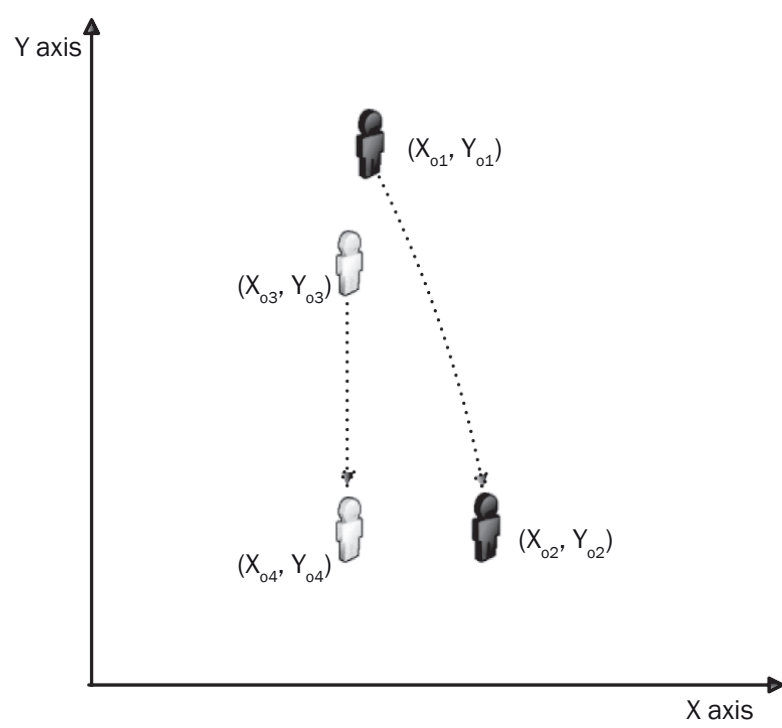

a) Data collection for overtaking behaviour

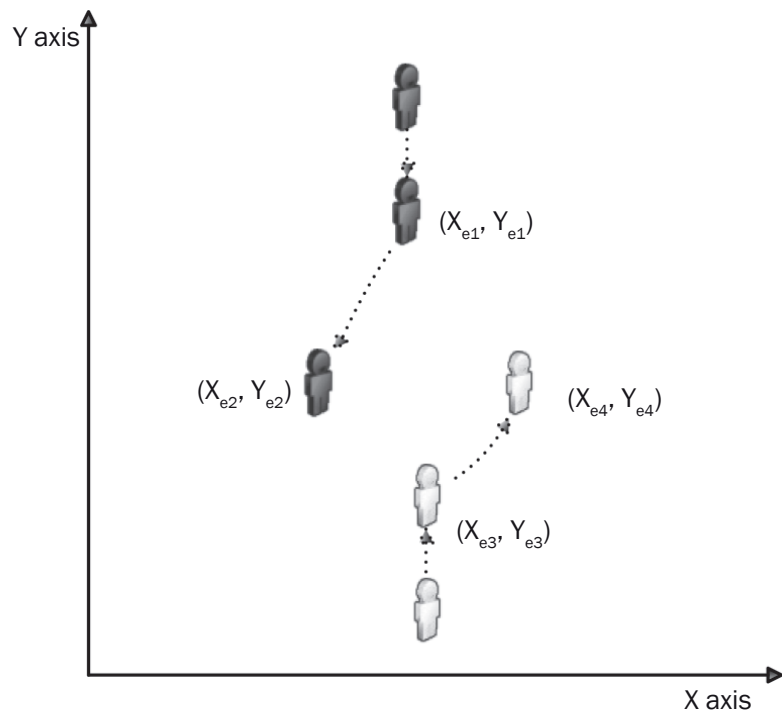

b) Data collection for evasive behaviour

Figure 2 - Data extraction of micro indicators

\section{MACRO CHARACTERISTICS OF PIBS}

\subsection{Occurrence intensities of PIBs}

The total observed time length for this study was 6.23 hours. During this period, the total number of overtaking movements was 1,609, and the total number of evasive movements was 1,395. The occurrence intensities of PIBs were calculated based on the above method. The scatter diagrams of the relationship between intensity and density are shown in Figure 3. It can be observed that, in general, the two scatter diagrams present a triangular shape. Both of the intensities increase firstly and then decrease with the increasing of density. A-E represents the different levels of service 
of pedestrian flow based on the primary measure of average space, the inverse of density, recommended in HCM 2010 [13].

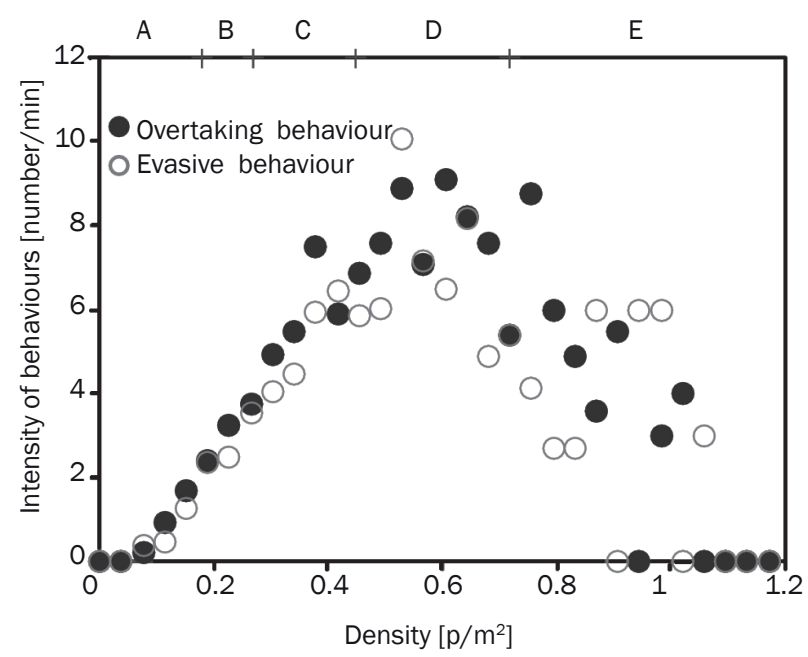

Figure 3 - Relationship between occurrence intensity and density

As can be seen in Figure 3, at extremely low density (less than $0.1 \mathrm{p} / \mathrm{m}^{2}$, LOS of A) or high density (larger than $1.1 \mathrm{p} / \mathrm{m}^{2}$, LOS of E nearly to F), almost none of PIBs could be observed. At the extremely low density, a walking pedestrian has no conflicts with other pedestrians resulting in that none of PIBs occur. On the contrary, at the extremely high density, pedestrians could not take an interactive behaviour due to the high restricted space, just forwarding others.

As the density increases, intensities of PIBs increase up to the maximum values at a certain density $\left(0.5-0.6 \mathrm{p} / \mathrm{m}^{2}\right.$, LOS of D). The increasing trend of intensities reflects that interactive effect among pedestrians is strengthening, and the demand for taking PIBs is rising. Meanwhile, there is enough available space for taking PIBs resulting in the increasing intensities. Density ranges from $0.1 \mathrm{p} / \mathrm{m}^{2}$ to $0.6 \mathrm{p} / \mathrm{m}^{2}$ and from LOS A to LOS D. It means that the level of pedestrian flow is changing from free walking conditions to interference with each other.

With the continuous increasing of density, the intensities of PIBs show a decreasing trend. The rapid decreasing trend of intensities is mainly due to the limited available walking space. Although demand for taking behaviours is very high, the restriction for taking PIBs among pedestrians is gradually becoming stronger. It means that from LOS D to LOS F, pedestrian flow condition is changing from interfering with each other to restraint among pedestrians.

Figure 4 shows the fitting curves to the results of relationship between the intensity of PIBs and density. It is worth noting that during the process of data collection, the observed time length at the density of $0.91 \mathrm{p} / \mathrm{m}^{2}$ is less than 30 seconds and one or two behaviours have been observed, resulting in high values of intensities (see Figure 3). Hence, the data for function fitting when density is larger than $0.91 \mathrm{p} / \mathrm{m}^{2}$ were ignored. The fitting equations are shown below ( $D$ is density):

$P_{0}=-32.44 \cdot D^{2}+37.72 \cdot D-3.21\left(R^{2}=0.86\right)$

$P_{\mathrm{e}}=-36.56 \cdot D^{2}+38.95 \cdot D-3.62\left(R^{2}=0.74\right)$

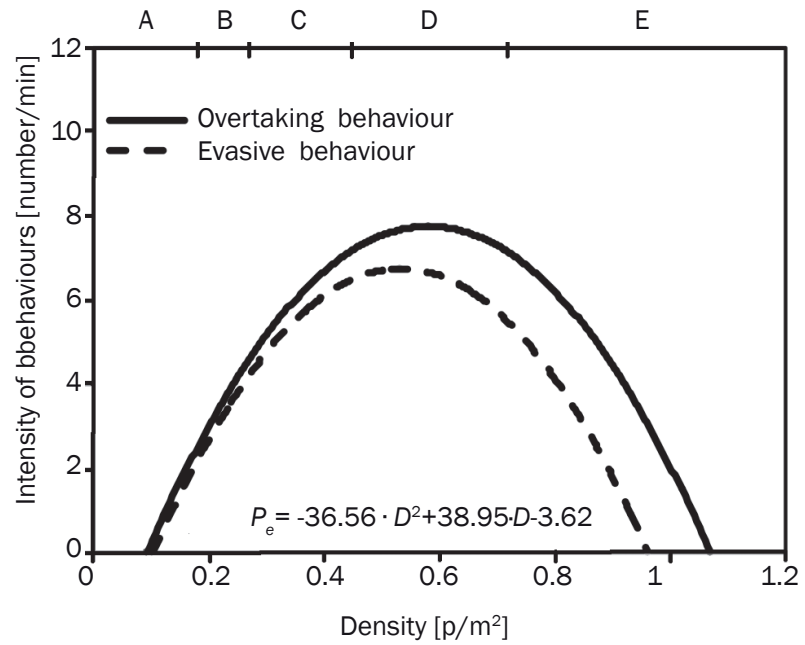

Figure 4 - Fitting curves to the two behaviours

\subsection{Characteristics of pedestrian sideways interactive behaviours}

As shown in Figures 3 and 4, two different densities may result in the same occurrence intensity of PIBs. However, difficulties of taking PIBs at the two different densities are obviously different. Proportions of pedestrian sideways interactive behaviours were calculated to illustrate this difference, as shown in Figure 5. A-E represents the different levels of service of pedestrian flow, recommended in HCM 2010 [13].

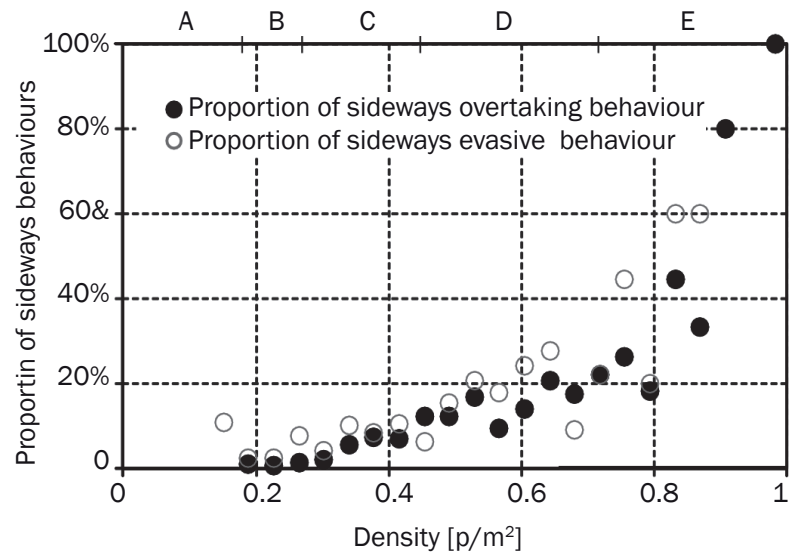

Figure 5 - Proportions of pedestrian sideways interactive behaviours 
Overall, the scatter diagrams to the proportions of sideways behaviours show a sustained increasing trend with the increasing of density. At the lowest density (less than $0.15 \mathrm{p} / \mathrm{m}^{2}$, LOS of $\mathrm{A}$ ), none of sideways behaviours occur; with the increasing of density (still less than $0.6 \mathrm{p} / \mathrm{m}^{2}$, from LOS B to LOS D), proportions of sideways behaviours are rising with low increasing rate; while when density is larger than $0.6 \mathrm{p} / \mathrm{m}^{2}$, the rate of increasing trend is high. At the highest density (LOS E, nearly LOS F), if a behaviour occurs, the behaviour is always a sideways behaviour because of the limited available walking space. For example, when the density is nearly $1 \mathrm{p} / \mathrm{m}^{2}$, all the observed overtaking movements are the sideways overtaking behaviour.

As can be seen in Figure 5, the scatter diagram of proportion of pedestrian sideways interactive behaviours could be divided into five parts: (0-0.2], none of sideways behaviours occur due to the large average space of each person; (0.2-0.4], conflicts among pedestrians begin to increase, sideways behaviours start to occur with the value of less than 10\%; (0.4-0.6], the proportion of sideways behaviours is from $10 \%$ to $20 \%$, and intensity of overtaking behaviour shows an increasing trend when the density is less than $0.6 \mathrm{p} /$ $\mathrm{m} 2 ;(0.6-0.8]$, the proportion of sideways behaviours is from $20 \%$ to $50 \%$, with the intensities of PIBs showing a decreasing trend; (0.8-1], the proportion of sideways behaviours is over $50 \%$ with rapid increase to $100 \%$.

\subsection{Differences of macro features between the two behaviours}

Although the overall occurrence intensity trend of overtaking behaviour shows similar characteristics to the trend of evasive behaviour, there were some differences between the two behaviours:

- Regarding overtaking behaviour, density with the maximum value of intensity is $0.61 \mathrm{p} / \mathrm{m}^{2}$, a little larger than the density of evasive behaviour ( 0.53 $\mathrm{p} / \mathrm{m}^{2}$ ). The observed maximum intensity of overtaking behaviour is 9.1 times per minute, while the value of evasive behaviour is 10 times per minute.

- Intensity of overtaking behaviour is always larger than the intensity of evasive behaviour. With the increasing of density, the gap at a certain density between the two behaviours slowly increases, as shown in Figure 4.

- According to Equations 1 and 2, the density of overtaking behaviour can be obtained where $P_{0}=0$, which is $1.07 \mathrm{p} / \mathrm{m}^{2}$, a little larger than the value of evasive behaviour $\left(0.96 \mathrm{p} / \mathrm{m}^{2}\right)$.

The reason for the above differences is that taking an evasive behaviour is a little more difficult than taking an overtaking behaviour at a certain density. It means that the space demand for taking an evasive behaviour is larger than the space demand for taking an overtaking behaviour. With the increasing of density, available space of evasive behaviour is more sensitive than the space of overtaking behaviour, which will be discussed in the next section. Besides, due to the same reason, the proportion of sideways evasive behaviour at a certain density is always larger than the proportion of sideways overtaking behaviour.

\section{MICRO CHARACTERISTICS OF PIBS}

\subsection{Micro characteristics of PIBs}

Micro indicators of PIBs are longitudinal distance before a behaviour occurs $(L)$ and horizontal distance after a behaviour has occurred $(H)$. The two distance indicators illustrate the amount of space necessary for taking behaviours to occur at a specific density. Because lots of PIBs had occurred, only some part of these behaviours' micro data were recorded. Descriptive statistical results about the two distance indicators are shown in Table 2. Data of $L$ and $H$ were collected separately, resulting in different sample sizes of $L$ and $H$.

Table 2 - Descriptive analysis for Micro characteristics of PIBs

\begin{tabular}{|c|c|c|c|c||}
\hline \multirow{2}{*}{$\begin{array}{c}\text { Descriptive } \\
\text { indexes }\end{array}$} & \multicolumn{2}{|c|}{$\begin{array}{c}\text { Overtaking } \\
\text { behaviour }\end{array}$} & \multicolumn{2}{c||}{ Evasive behaviour } \\
\cline { 2 - 5 } & $L_{o}$ & $H_{o}$ & $L_{e}$ & $H_{e}$ \\
\hline \hline Sample size & 147 & 97 & 196 & 269 \\
\hline Mix [m] & 0.3 & 0.45 & 0.15 & 0.3 \\
\hline Mean [m] & 1.01 & 0.74 & 3.08 & 0.69 \\
\hline Max [m] & 2.35 & 1.25 & 5.95 & 1.35 \\
\hline $\begin{array}{c}\text { Standard } \\
\text { deviation [m] }\end{array}$ & 0.45 & 0.18 & 1.14 & 0.18 \\
\hline $\begin{array}{c}\text { Range of } \\
\text { density } \\
{\left[\mathrm{p} / \mathrm{m}^{2}\right]}\end{array}$ & $0.11-$ & $0.07-$ & $0.076-$ & $0.11-$ \\
0.76 & 0.79 & 0.95 \\
\hline \hline
\end{tabular}

As shown in Table 2, the distance indicators were also varied with density. For example, the value of $L_{0}$ ranged from $0.3 \mathrm{~m}$ to $2.35 \mathrm{~m}$. At the beginning of behaviours, a minimum of longitudinal distance of overtaking behaviour is $0.3 \mathrm{~m}$, and the distance of evasive behaviour is $0.15 \mathrm{~m}$. The reason for these small longitudinal distances when the behaviours occur is that a pedestrian can urgently decelerate their walking speed and change their walking direction with a large angle during the walk. Furthermore, the minimum value of $H_{e}$ is $0.3 \mathrm{~m}$, and it is reasonable that both of the two related pedestrians take the sideways evasive behaviours to avoid conflicts.

The scatter diagrams of the relationship between these two distance indicators and density are shown in Figure 6. Overall, the density has no significant effect on the longitudinal and horizontal distances at low density until the threshold value; with the continuous 
increasing of density, both $L$ and $H$ show a decreasing trend.

To determine the threshold values for the two behaviours and two distance indicators, the method of one by one to calculate the correlations between these two distance indicators and density has been adopted. The density is less than $0.07 \mathrm{p} / \mathrm{m}^{2}$, $0.11 \mathrm{p} / \mathrm{m}^{2}, 0.15 \mathrm{p} / \mathrm{m}^{2} \ldots$, until there is a significant correlation between these two distance indicators and density. When density is larger than the threshold value, linear regression is selected to show the relationship between the micro indicators and density with low adjusted $R^{2}$ (less than 0.2), as shown in Figures $6 a$ and $6 b$. Regarding pedestrian overtaking behaviour, both density threshold values $L_{o}$ and $H_{o}$ are $0.34 \mathrm{p} / \mathrm{m}^{2}$. Regarding pedestrian evasive behaviour, the density threshold value $L_{e}$ is $0.26 \mathrm{p} / \mathrm{m}^{2}$, a little less than the density threshold value of $H_{e}\left(0.34 \mathrm{p} / \mathrm{m}^{2}\right)$. The fitting equations are shown in each chart when density is larger than the density threshold value.
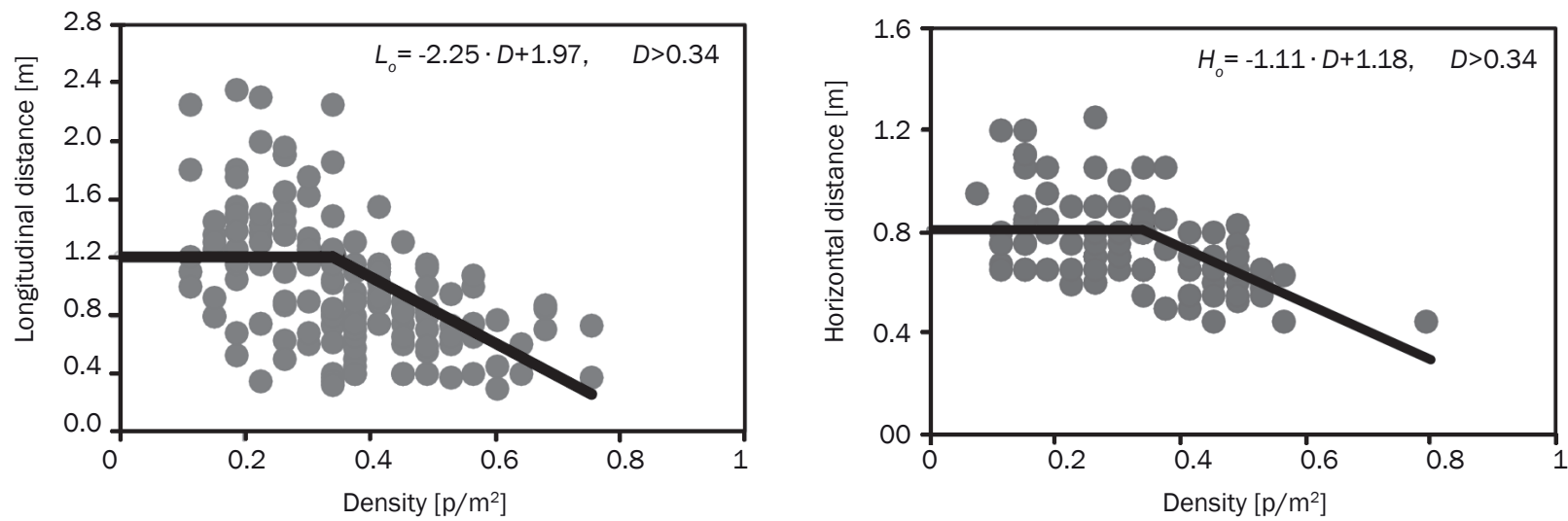

a) Micro characteristics of pedestrian overtaking behaviour
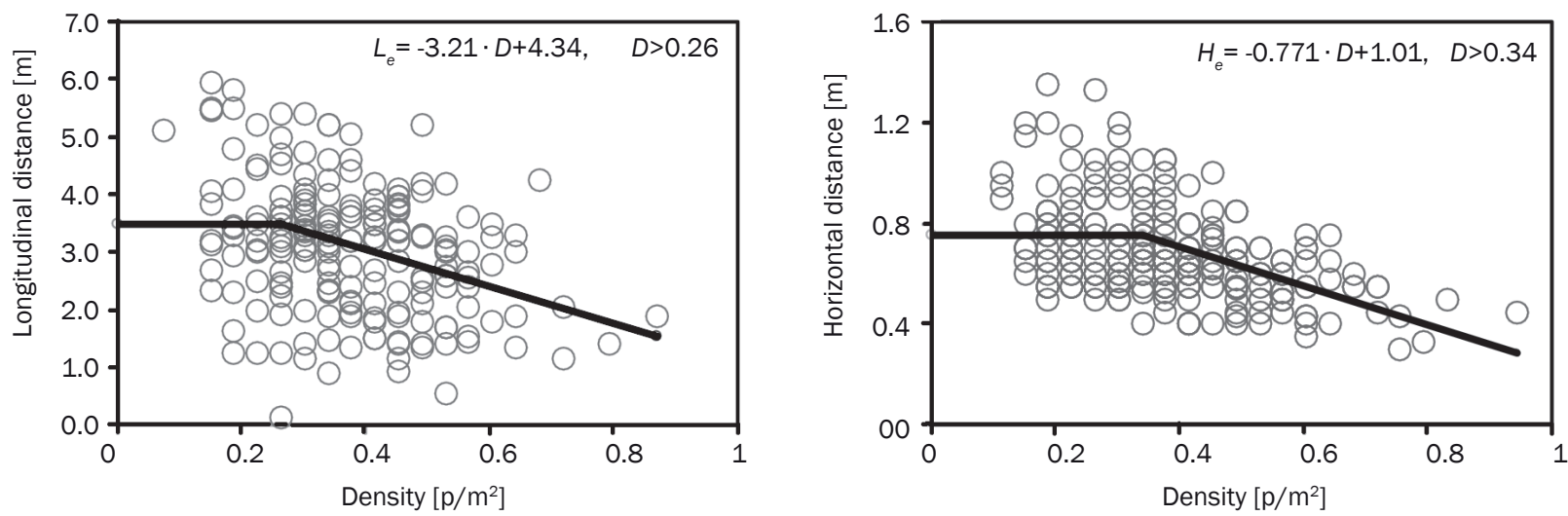

b) Micro characteristics of pedestrian evasive behaviour
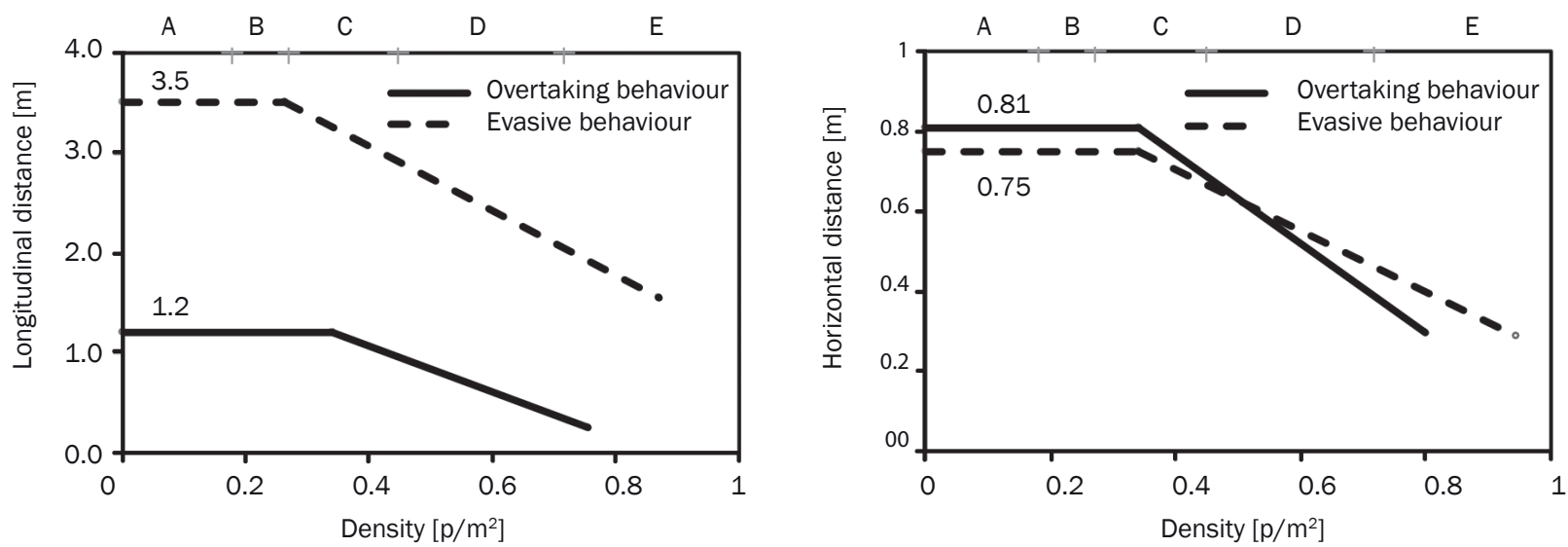

c) Micro characteristics of pedestrian interactive behaviours

Figure 6 - Relationship between the micro indicators of PIBs and density 
Figure $6 \mathrm{c}$ shows the differences of micro characteristics between the two comparative interactive behaviours. The following results could be found:

- Regarding the horizontal distances of PIBs $(H)$, both of the density threshold values of the two behaviours are $0.34 \mathrm{p} / \mathrm{m}^{2}$. And the distance values of the two different behaviours appear similar characteristics at low density. With the continuous increasing of density, both of the horizontal distances show a decreasing trend.

- Regarding the longitudinal distances before the behaviours occur $(L)$, the density threshold value of evasive behaviour $\left(0.26 \mathrm{p} / \mathrm{m}^{2}\right)$ is less than the density value of overtaking behaviour $\left(0.34 \mathrm{p} / \mathrm{m}^{2}\right)$. The value of $L_{e}$ is about $3.5 \mathrm{~m}$ at low density, almost three times larger than the value of $L_{0}(1.2$ $\mathrm{m})$. Therefore, the difference of micro features between the two behaviours mainly results from the difference between $L_{e}$ and $L_{o}$. It is worth noting that the decreasing rate of $L_{e}$ is a little quicker than the decreasing rate of $L_{o}$, indicating that $L_{e}$ is more sensitive than $L_{o}$ with the increasing of density.

- To sum up, space supply for taking behaviours initially keeps a certain value and then decreases with the increasing of density. Density threshold values of different behaviours and distance indicators correspond to LOS C (except the density threshold value of $L_{e}$, at LOS B, nearly LOS C). Space requirement for evasive behaviour is larger than the space for overtaking behaviour. In addition, distance values of $L_{o}$ and $H_{0}$ at low density are $1.2 \mathrm{~m}$ and 0.75 $\mathrm{m}$, which indicates the personal space requirement consistent with previous study results [17-19].

\subsection{Relationship between the macro and micro characteristics of PIBs}

To understand the relationship between macro and micro characteristics of PIBs, the method of supply-demand analysis was adopted. Demand means that conflicts among pedestrians increase rapidly with the increasing of density. Supply means the available space for taking behaviours to occur at a certain density. Therefore, the occurrence intensity features are a process of interaction between behaviours' demand and space supply at different densities.

When density increases from zero to density threshold values of $L\left(0.34 \mathrm{p} / \mathrm{m}^{2}\right.$ to overtaking behaviour, and $0.26 \mathrm{p} / \mathrm{m}^{2}$ to evasive behaviour, LOS A to LOS C), the conflicts among pedestrians increase with enough space supply for taking behaviours; hence occurrence intensities of PIBs increase rapidly. When the density continues to increase from the density threshold value to the densities of maximum intensities $\left(0.61 \mathrm{p} / \mathrm{m}^{2}\right.$ for overtaking behaviour, and $0.53 \mathrm{p} / \mathrm{m}^{2}$ for evasive behaviour, LOS C to LOS D), space supply for behaviours shows a decreasing trend with sufficient space for taking behaviours leading to the slow growth of intensities. When density is larger than the densities of maximum intensities (LOS D to nearly LOS F), even though demand for taking behaviours is high, the intensities begin to decrease until zero due to the restriction of space supply, along with many sideways behaviours.

Moreover, space requirement for evasive behaviour is larger than the space requirement for overtaking behaviour at a certain density thus, the density of maximum intensity and the micro density threshold value of evasive behaviour are lower than the densities of overtaking behaviour.

\section{REVISED PEDESTRIAN WALKWAY LOS AND DISCUSSION}

The above sections have analysed the characteristics of PIBs. The results of these features can provide some critical values to explain the veracity of LOS description presented in HCM2010, as shown in the left part of Figure 7. The threshold values of HCM2010 is based on the flow-density chart, with qualitative description of the conflicts among pedestrians. The critical densities shown in the right part of Figure 7 reflect the actual features of PIBs at different densities. Some inaccurate descriptions could be identified in HCM2010. For example, the qualitative description at LOS $\mathrm{C}$ is that space is sufficient for overtaking behaviour, while density with the maximum intensities of overtaking behaviour is $0.61 \mathrm{p} / \mathrm{m}^{2}$ and corresponds to LOS D.

A revised pedestrian walkway LOS in China has been proposed based on the characteristics of PIBs at different densities, as shown in the right part of Figure 7. At LOS A, density is less than $0.19 \mathrm{p} / \mathrm{m}^{2}$ without conflicts among pedestrians; at LOS B (density from $0.19 \mathrm{p} / \mathrm{m}^{2}$ to $0.34 \mathrm{p} / \mathrm{m}^{2}$ ), pedestrians can take overtaking behaviours freely; at LOS C (density from $0.34 \mathrm{p} / \mathrm{m}^{2}$ to $0.61 \mathrm{p} / \mathrm{m}^{2}$ ), space is sufficient for overtaking behaviour up to the maximum intensity of PIBs; at LOS D (density from $0.61 \mathrm{p} / \mathrm{m}^{2}$ to $0.80 \mathrm{p} / \mathrm{m}^{2}$ ), the proportion of sideways behaviours shows an increasing trend due to the lack of sufficient space; when density is larger than $0.80 \mathrm{p} / \mathrm{m}^{2}$; at LOS $\mathrm{E}$, the proportion of sideways behaviours increases rapidly while intensity of PIBs decreases to zero; at LOS F (density $>1.07$ $\left.\mathrm{p} / \mathrm{m}^{2}\right)$, none of PIBs could be observed, and forward progress is made only by shuffling. Therefore, the revised pedestrian walkway LOS is consistent with the description presented in HCM2010.

With the rapid development of rail transit in China, the metro station walkways have been designed to guide the pedestrian flow. The rail transit management department needs a standard to evaluate LOS of these facilities. The traditional method was based on the relationship between pedestrian flow and density. However, during the process of pedestrians 


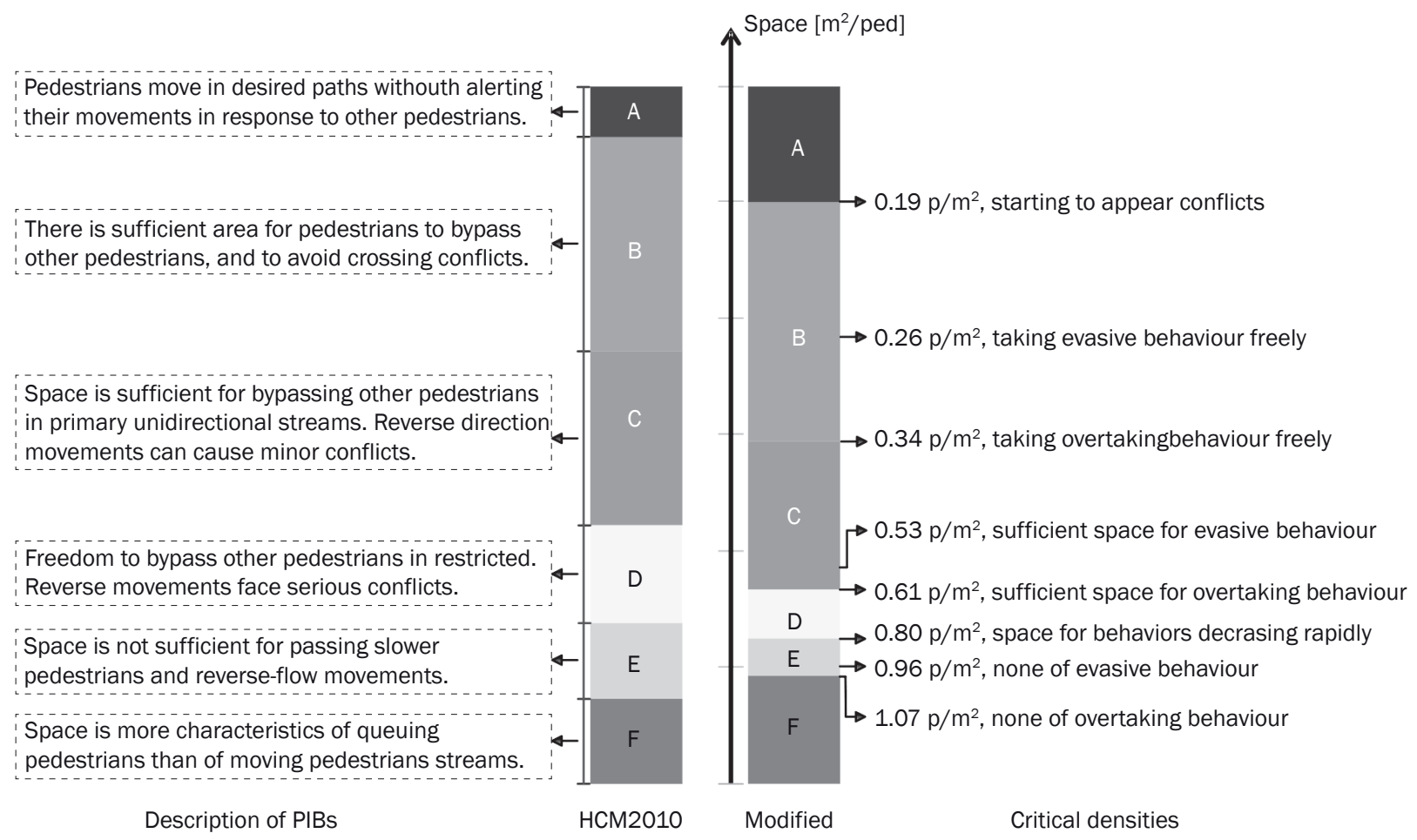

Figure 7 - Description of PIBs in HCM2010 and the revised LOS

moving in clusters, the common interactive behaviours will inevitably happen, and their characteristics can show pedestrian comfort level on the walking condition. Compared to the original LOS presented in HCM2010, our revised LOS reflects the real pedestrian subjective perception at different densities, which can be used to evaluate the LOS on metro station walkways in China.

Furthermore, the effect of PIBs has been considered in the study of pedestrian simulation modelling [15-16]. Micro indicators of PIBs can be used as pedestrian kinematic parameters incorporated in these models, and model calibration can be based on the macro features of PIBs.

\section{CONCLUSIONS}

This paper explored the characteristics of PIBs in order to revise the LOS on metro station walkways in China. Macro and micro indicators of PIBs were calculated based on field observations. Macro features of overtaking and evasive behaviours were analysed, the occurrence intensity and proportion of sideways behaviours named. The differences of macro features between the two behaviours were also discussed. Micro indicators of PIBs were analysed at the different levels of service. Besides, the relationship between macro and micro characteristics of PIBs were explored. Finally, a revised LOS on metro station walkways was proposed.
The results showed that the occurrence intensities of PIBs initially increased with moderate density and later decreased with high density that reduced the available space. The proportions of sideways behaviours showed an increasing trend from zero to $100 \%$ with the increasing of density. The differences between overtaking behaviour and evasive behaviour are the density values of maximum intensities and the gap of occurrence intensity between them at a certain density.

Micro distance indicators which illustrate the amount of space necessary for taking behaviours to occur at a specific density first kept a certain value and then decreased with the increasing of density. The density threshold value of $L_{e}$ was less than the value of $L_{o}$. The density threshold value of $H_{e}$ was the same as the value of $H_{0}$. Occurrence intensity features of PIBs mainly resulted from micro characteristics. With our results, the critical densities based on the macro and micro characteristics of PIBs were discussed with the qualitative description at different levels of service presented in HCM2010. Finally, a revised pedestrian walkway LOS was proposed based on the macro and micro characteristics of PIBs in China.

In our study, there are some limitations. During the process of micro data extraction the pedestrians' coordinates could be affected by other persons resulting in small sample size at high density. The method of automatic video extraction could be developed to solve this problem in a future study. The data of PIBs at 
different walking facilities should be collected, such as different widths of walkways, the stairs, and so on. Besides, the influence of personal features was ignored, such as the gender, age and the condition of carry-on luggage. The authors recommend that the future research should focus on these issues.

\section{ACKNOWLEDGEMENTS}

The authors are grateful to three anonymous referees for their constructive comments and suggestions to improve the quality and clarity of this paper. This research was supported by the National Science Foundation of China (No. 5108342).

\section{单肖年 ${ }^{1}$, 叶建红 ${ }^{1}$ (通讯作者), 陈小鸿 ${ }^{1}$ \\ 1 同济大学 道路与交通工程教育部重点实验室, 上 海 201804}

\section{基于行人交互行为特征的水平通道服务水平修正方法}

\section{摘要}

本文的研究目标是分析行人交互行为特征以对地 铁站水平通道行人流服务水平进行修正。基于上海 市地铁站水平通道采集的数据, 计算了行人超越及 避让行为宏微观特征指标。两种交互行为的发生强 度在低密度下首先呈现增长的趋势, 随后高密度下 由于可实施空间的不足呈现下降的趋势。论文讨论 了不同服务水平下的侧身交互行为特征以表征不同 密度下行人交互行为发生的难易程度。我们发现行 为实施的可利用空间是导致行为强度特征的主要因 素。此外，不同行为可实施空间的差异性是引起两 种交互行为差异性的原因。最后，基于行人交互行 为宏微观特征, 提出了修正的水平通道行人流服务 水平。

\section{关键词}

行人服务水平; 超越行为; 避让行为; 宏微观特征; 地铁站水平通道

\section{REFERENCES}

[1] Tatebe K, Nakajima H. Avoidance behaviour against a stationary obstacle under single walking: A study on pedestrian behaviour of avoiding obstacles (I) [in Japanese]. Journal of Architecture Planning and Environmental Engineering. 1990;418:51-57.

[2] Kitazawa K, Fuijyama T. Pedestrian vision and collision avoidance behaviour: investigation of the information process space of pedestrians using an eye tracker. In: Klingsch WWF, Rogsch C, Schadschneider A, Schreckenberg M, editors. Pedestrian and Evacuation Dynamics 2008. London, UK: Springer, 2010. p. 95-108.

[3] Fang ZM, Song WG, Liu X, et al. A continuous distance model (CDM) for the single-file pedestrian movement considering step frequency and length. Physica A. 2012;391(1-2):307-316.
[4] Wu JR, Feng JD, Lu SG. Research on pedestrian overtaking behaviour in passageway. Journal of Tongji University (Natural Science). 2012;40(2):228-234.

[5] Miyazaki K, Matsukura H, Katuhara M, et al. Behaviours of pedestrian group overtaking wheelchair user. Tokyo: National Maritime Research Institute (NMRI). Report 181-004; 2004.

[6] Wolff M. Notes on the behaviour of pedestrians. In: Birenbaum $A$, editor. People in places: the sociology of the familiar. London, UK: Nelson Press; 1973.

[7] Kim S, Choi J, Kim S, et al. Personal space, evasive movement and pedestrian level of service. Journal of Advanced Transportation. 2014;48(6):673-684.

[8] Shan XN, Ye JH, Chen XH. Critical walking space requirement for collision avoidance of pedestrians: an experimental study. Proceedings of the 14th COTA International Conference of Transportation Professionals (CICTP 2014); 2014 July 4-7; Changsha, China; 2014. p. 2369-2380.

[9] Wu JR, Lu SG. Feature analysis and operation evaluation of pedestrian weaving zone. Journal of the Transportation Research Board. 2013;2393:66-74.

[10] Sun LS, Yang ZF, Rong J, et al. Study on the weaving behaviour of high density bidirectional pedestrian flow. Mathematical Problems in Engineering. 2014; Article ID 765659, 9 p.

[11] Ma J, Song W, Fang Z, et al. Experimental study on microscopic moving characteristics of pedestrians in built corridor based on digital image processing. Building and Environment. 2010;45:2160-2169.

[12] Fruin JJ. Pedestrian planning and design. Mobile: Elevator World; 1990.

[13] Highway Capacity Manual 2010. Washington DC: Transportation Research Board, National Research Council (TRB Special Report 616).

[14] Highway Capacity Manual 2000. Washington DC: Transportation Research Board, National Research Council (TRB Special Report 209).

[15] Yuen JKK, Lee EWM. The effect of overtaking behaviour on unidirectional pedestrian flow. Safety Science. 2012;50(8):1704-1714.

[16] Gotoh H, Harada E, Andoh E. Simulation of pedestrian contra-flow by multi-agent DEM model with self-evasive action model. Safety Science. 2012;50(2):326-332.

[17] Dabbs J, Stokes N. Beauty is power: the use of space on the sidewalk. Sociometry, 1975;38(4):551-557.

[18] Burgess J. Interpersonal spacing between surrounding nearest neighbors reflects both familiarity and environmental density. Ethology and Sociobiology. 1983;4(1):11-17.

[19] Willis A, Gjersoe N, Havard C, et al. Human movement behaviour in urban spaces: implications for the design and modeling of effective pedestrian environments. Environment and Planning B: Planning and Design. 2012;31(6):805-828. 